\title{
ON MODERATE DEVIATIONS FOR COUNT DATA AND GAMMA APPROXIMATION
}

\author{
QINGWEI LIUD
}

(Received 17 December 2021; first published online 8 February 2022)

\section{Mathematics subject classification: primary 60F05; secondary 60F10.}

Keywords and phrases: Stein's method, translated Poisson approximation, gamma approximation, size biasing, zero biasing, moderate deviation.

The main goal of this thesis is to study moderate deviations for the distributions of count data. In the first part of this thesis, we use the distribution of the number of records to demonstrate that the right tail probabilities of counts of rare events are generally better approximated by the right tail probabilities of the Poisson distribution than those of the normal distribution. We then show that the moderate deviation in Poisson approximation generally requires an adjustment and, with a suitable adjustment, we establish a better error estimate of the moderate deviation in Poisson approximation than those in [1].

We then focus on the moderate deviation in negative binomial approximation for over-dispersed count data. Two theorems are established: one considers the sum of locally dependent nonnegative integer-valued random variables and the other compares a coupling of its size-biased and zero-biased distributions.

The next part of the thesis focuses on gamma approximation. We then show that a nonnegative random variable follows a gamma distribution if and only if its size biasing equals its zero biasing. We combine this characterisation with Stein's method to establish simple bounds for gamma distribution approximation to the sum of nonnegative independent random variables, a class of compound Poisson distributions and the negative binomial sum of random variables.

A classical result in renewal theory is the central limit theorem for renewal processes. Because the number of renewals is integer-valued and is more naturally approximated by a translated Poisson, an estimate of the distance between the number of renewals and a suitable translated Poisson is desirable. We finally provide an explicit bound for the number of renewals under the Wasserstein distance using Stein's method.

Thesis submitted to the University of Melbourne in July 2021; degree approved on 12 October 2021; supervisors Aihua Xia and Shaunming Li.

(C) The Author(s), 2022. Published by Cambridge University Press on behalf of Australian Mathematical Publishing Association Inc. 


\section{Reference}

[1] L. H. Chen, X. Fang and Q.-M. Shao, 'Moderate deviations in Poisson approximation: a first attempt', Statist. Sinica 23 (2013), 1523-1540.

QINGWEI LIU, School of Physical and Mathematical Sciences,

Nanyang Technological University, Singapore

e-mail: xiaozong30@gmail.com 